\title{
Universidad, multiculturalidad, interculturalidad y sociedad: Desafíos del acompañamiento comu- nitario intercultural desde la URACCAN
}

\author{
University, multiculturalism, interculturality and society: Challenges of intercultural community \\ accompaniment from URACCAN
}

Yuri Hamed Zapata Webb' ${ }^{1}$

\section{Resumen}

En este artículo se ha abordado la relación existente entre las concepciones de universidad, multiculturalidad, interculturalidad y sociedad, desde la perspectiva del acompañamiento comunitario intercultural que se plantea desde la práctica de la Universidad de las Regiones Autónomas de la Costa Caribe Nicaragüense (URACCAN), , que concibe este acompañamiento como una de sus funciones institucionales en aras del desarrollo con identidad, el buen vivir de los pueblos y la construcción de ciudadanías interculturales.

Se ha realizado un recorrido básico sobre cada concepto tratado, para encausarnos en el entendimiento de los retos que significa el desarrollo e implementación del acompañamiento comunitario intercultural en contextos multiculturales, en función de asegurar que los pueblos puedan elevar sus propias voces en términos de su bienestar físico, material y espiritual. El artículo obedece a una investigación con una visión cualitativa, que parte de un análisis documental conceptual y analítico sobre lo que comprende el acompañamiento comunitario Intercultural, desde la visión de URACCAN.

Este acompañamiento al desarrollo comunitario con una visión intercultural enfrenta sus desafíos desde su conceptualización e interiorización en las instituciones de educación superior y la lógica de su articulación al desarrollo comunitario.

Palabras clave: Universidad; Universidad Comunitaria Intercultural; multiculturalidad; interculturalidad; acompañamiento comunitario intercultural; extensión social.

\section{Abstract}

This article has addressed the relationship between the conceptions of university, multiculturalism, interculturality and society, from the perspective of intercultural community support that arises from the practice of the University of the Autonomous Regions of the Nicaraguan Caribbean Coast (URACCAN), which conceives this accompaniment as one of its institutional functions for the sake of development with identity, the good living of the peoples and the construction of intercultural citizenships.

A basic tour has been carried out on each concept dealt with, to guide us in understanding the challenges that the development and implementation of intercultural community accompaniment in multicultural contexts means, in order to ensure that peoples can raise their own voices in terms of their well-being physical, material and spiritual. The article follows an investigation with a qualitative vision, which starts from a conceptual and analytical documentary analysis on what is comprised of Intercultural community accompaniment, from the URACCAN perspective.

\footnotetext{
1 Candidato Doctoral, Vicerrector General URACCAN, yuri.zapata@uraccan.edu.ni ; https://orcid.org/0000-0002-0209-4843
}

Recibido: 05/03/2020 - Aprobado: 06/19/2020

Zapata Webb, Y. (2020). Universidad, multiculturalidad, interculturalidad y sociedad: Desafíos del acompañamiento comunitario intercultural desde la URACCAN. Revista Universitaria Del Caribe, 25(02), 44 - 51. https://doi.org/10.5377/ruc.v25i02.10474 
This accompaniment to community development with an intercultural vision faces its challenges from its conceptualization and internalization in higher education institutions and the logic of its articulation to community development.

Keywords: University; Intercultural Community University; multiculturalism; interculturality; intercultural community accompaniment; social extension.

\section{Introducción}

Abya Yala para nuestros pueblos, América Latina para quienes nos trataron de conquistar, dominar y colonizar; es un área geográfica donde habitan 42 millones de personas de pueblos y nacionalidades originarios, así como 120 millones afrodescendientes que, en su conjunto representan más del $28 \%$ de la población de la región ${ }^{2}$. Esto nos da un alto carácter de la diversidad cultural y la multiculturalidad, presente en los espacios territoriales llamados países.

Hoy cada uno de nuestros países, reconoce desde sus marcos normativos, la presencia "ecología" como lo señala De Sousa Santos, de la diversidad cultural, de sistemas de conocimientos, de saberes y haceres, lo cual se suma a la creciente demanda de la educación superior para el desarrollo y fortalecimiento de las sociedades, en donde la extensión social universitaria, la vinculación universidad-sociedad, el acompañamiento comunitario intercultural juegan un rol importante en la construcción de las comunidades modernas.

El presente artículo es un proceso de reflexión sobre la extensión social, extensión universitaria, proyección social, acción social, vinculación social, acompañamiento comunitario intercultural, tal y como la llamamos en nuestros marcos normativos de países y de las instituciones de educación superior: leyes, estatutos, políticas, Reglamentos, manuales, entre otros. Siguiendo la lógica de los procesos de creación, recreación de conocimientos, saberes, sentires, haceres y quereres de los pueblos; el ensayo hace una contraposición entre preguntas y desafíos que tiene el acompañamiento comunitario intercultural; visto desde URACCAN como una función integradora de la gestión institucional. Tal y como lo plantea la hermenéutica; se trata de dar salida a una visión de conjunto para responder ante las realidades presentes.

Esta reflexión colectiva, trata de articular los conceptos que nos han traído a este espacio de socialización, de preocupación hacia dónde nos van a llevar los acontecimientos mundiales, sociales y naturales que, de una u otra manera nos afectan como pueblos y como instituciones. Hablar de universidad, multiculturalidad, interculturalidad y sociedad es un reto grande, al imbricar elementos que no son comunes, pero que tienen una intrínseca relación entre ellos: La universidad es parte de la sociedad, la sociedad es multicultural y se busca la interculturalidad como constructo social educativo; por lo tanto, es preciso trabajar con cada uno de ellos de manera que podamos concebir, interiorizar y después actuar en términos de la implementación de acciones institucionales para el desarrollo con identidad, la autonomía territorial, el buen vivir de los pueblos y la construcción de ciudadanías interculturales en una sociedad de paz.

La presente reflexión está constituida por una breve reseña de lo que es la URACCAN, realiza un acercamiento básico sobre los conceptos de multiculturalidad, interculturalidad, la concepción sobre lo que es el acompañamiento comunitario intercultural. Las conclusiones reflejan que es necesario trabajar por sociedades justas, equitativas, inclusivas, interculturales que permitan construir ciudadanías interculturales. En este sentido los procesos de acompañamiento comunitario intercultural que desde las instituciones llevamos acabo, deben apuntar desde una visión holística a considerar las realidades de los pueblos en su globalidad, para ayudarlos a construir un mundo más equitativo, justo, inclusivo, solidario e intercultural.

De allí que se debe ver esta función institucional, como el instrumento dónde se aborde el multiculturalismo y su transformación en un sistema relacional intercultural, que permita transformaciones sociales profundas desde el trabajo que desarrolla la universidad.

2 Mato, D. 2017, Educación Superior y Pueblos Indígenas y Afrodescendientes en América Latina; Políticas y Prácticas de Inclusión, Democratización e Interculturalización. EDUNTREF, 1 Edición, Buenos Aires, Argentina. 


\section{La URACCAN}

La URACCAN, como institución de educación superior en contextos multiculturales y de autonomía regional multiétnica, es una universidad comunitaria intercultural ubicada en el Caribe de Nicaragua, es miembro del Consejo Nacional de Universidades (CNU), su fundación data de 1994, su origen parte de la necesidad de desarrollar un nuevo modelo de educación superior que sirva como instrumento del verdadero empoderamiento de los pueblos afrodescendientes, indígenas, mestizos costeños y otros pueblos, con un paradigma educativo a través del cual los estudiantes pueden aprender desde la creación, recreación de sus conocimientos, saberes, haceres, donde sus identidades, lenguas, culturas, espiritualidades, historias son parte de sus propios procesos educativos.

La URACCAN tiene el propósito de dar oportunidad e incluir a quienes históricamente han estado excluidos en los procesos del desarrollo individual y colectivo. Se ha constituido de esa manera en un espacio de diálogo, de concertación y búsqueda para dar solución a los problemas que presentan los pueblos y sus culturas, la sociedad y el Estado-Gobierno.

\section{Un acercamiento conceptual}

\section{Universidad}

Todos conocemos y sabemos que, las universidades desde sus orígenes han desempeñado el rol del desarrollo del pensamiento crítico, el de consciencia social para la construcción de un mundo justo, equitativo, inclusivo, pertinente y que genera solución a los problemas para cada contexto natural y social. Sin embargo, en el camino se ha fragmentado esta visión, por una más mercantilizada, más burócrata, más colonizadora al reproducir patrones de un sólo sistema de conocimiento el que cual denominamos occidental y que discrimina lo "otro" en el supuesto que no cumple con "lo científico". De ahí que en las conferencias Regionales de Educación Superior realizadas en La Habana, Cuba, en 1996; en Cartagena de Indias, Colombia en 2008 y en Córdoba, Argentina en 2018; donde se ratifica a la educación superior como un bien social, un bien público, se ha señalado enfáticamente que, las IES deben de repensarse para atender la diversidad de matrices culturales. Ese es otro de nuestros grandes desafíos, el cómo atender la diversidad desde cada una de nuestras responsabilidades y razón de ser como centro de cultivo y cosecha del conocimiento.

De igual manera se ha sintetizado en la CRES 2018, que:

El conocimiento constituye un bien social, colectivo y estratégico al que deben tener acceso todas las personas sin distinción social, género, etnia ni religión, y el Estado debe garantizar su acceso y democratización a toda la sociedad" Pero, también refiere desde el 2008, que: en América Latina y el Caribe, particularmente, se necesita una educación que contribuya eficazmente a la convivencia democrática, a la tolerancia y a promover un espíritu de solidaridad y de cooperación; que construya la identidad continental; que genere oportunidades para quienes hoy no las tienen y que contribuya, con la creación del conocimiento, a la trasformación social y productiva de nuestras sociedades.

En honor a la misión sustantiva de la universidad, las CRES (2008 y 2018) han ratificado que:

es necesario promover el respeto y la defensa de los derechos humanos, incluyendo: el combate contra toda forma de discriminación, opresión y dominación; la lucha por la igualdad, la justicia social, la equidad de género; la defensa y el enriquecimiento de nuestros patrimonios culturales y ambientales; la seguridad y soberanía alimentaria y la erradicación del hambre y la pobreza; el diálogo intercultural con pleno respeto a las identidades; la promoción de una cultura de paz, tanto como la unidad latinoamericana y caribeña y la cooperación con los pueblos del Mundo. 


\section{Multiculturalidad e interculturalidad}

Con referencia al carácter pluricultural, multicultural, multilingüe de nuestra región, tenemos que acotar los términos de la multiculturalidad e interculturalidad.

Hay muchos escritos respecto a lo que es la multiculturalidad y lo que ello ha conllevado a coincidencias entre los autores que, refiere la existencia de varias culturas que conviven en un mismo espacio físico, geográfico o social, sin que se de una interacción de doble vía. Muchas veces, la ausencia de interacción y comunicación intercultural es fuente de tensiones y conflictos fuertes. Pero, lo importante de esto es que, si se logra desarrollar un proceso de intercambio con respeto y tolerancia; entonces, se pasa a una situación intermedia que se conoce como el principio del multiculturalismo. Este comprende la aceptación de las formas diversas que existen de las diferencias culturales, conocidas como la política de la identidad, la política de la diferencia o política del reconocimiento.

Muchas veces se confunde la multiculturalidad con la interculturalidad; si bien es cierto, que como ya se ha dicho, la primera comprende la convivencia de dos o más culturas en un área particular. Sin embargo, cuando hablamos de interculturalidad, de acuerdo a Hooker (2019):

muchas veces, se hace desde el discurso, sin realmente profundizar en los grandes desafíos que presenta, tales como: compartir el poder en los diferentes espacios de toma de decisiones, propiciar la igualdad de las oportunidades, la redistribución equitativa de los recursos, la visibilización y conquista de espacios negados en lo histórico, social, político, cultural y ambiental de los diferentes pueblos; así como su visibilización en las estadísticas nacionales y la puesta en práctica de políticas y acciones con y para los pueblos que partan de sus verdaderas necesidades y prioridades.

Desde la UNESCO (2006), la interculturalidad se concibe como presencia e interacción equitativa de diversas culturas; la posibilidad de generar expresiones culturales de enseñanzas compartidas a través del diálogo y el respeto mutuo.

Para las universidades indígenas, interculturales y comunitarias, la interculturalidad comprende el desarrollo de procesos de toma de decisiones conjuntas y concertadas en igualdad de condiciones. Su finalidad es la promoción y práctica de la equidad, la inclusividad, el respeto, la comprensión, la aceptación mutua y la creación de sinergias para el establecimiento de sociedades no discriminatorias, libres de racismo3.

Esto es resignificar la vocación de paz; con lo que se puede apostar al derecho de la construcción de ciudadanías interculturales, el desarrollo con identidad y el buen vivir de los pueblos.

\section{Acompañamiento comunitario intercultural: Una mirada diferente a la extensión social}

\section{Extensión social ${ }^{4}$}

En el proceso de desarrollo de las universidades Centroamericanas, se ha concebido a la extensión social de acuerdo a 4 dimensiones:

Por su naturaleza: Es el proceso universitario transversal, que se expresa a través de acciones de cooperación e intercambio del conocimiento y creatividad de estudiantes, docentes e investigadores con la sociedad.

Por su contenido: Es la coordinación desde la investigación y el postgrado, atienden la misión sociocultural y aportan a la solución de problemas, enriquecen el currículo y la formación integral.

\footnotetext{
3 Parafraseo a Hooker, 2019.

4 SICAUS-CSUCA (2019). Informe de Gestión de las Acciones Relacionadas con la Agenda de trabajo del SICAUS, 19 Agosto, Managua, Nicaragua.
} 
Por su procedimiento: Es el aprovechamiento racional de los recursos y potencialidades de las comunidades basadas en modelos interculturales.

Por su finalidad: Es la contribución al desarrollo y transformación hacia una sociedad sostenible.

En este marco, Benítez (2016) refiere que, sólo a través de la extensión universitaria se puede trabajar y articular el desarrollo local con el nacional, se contribuye de esta manera al buen vivir de los pueblos. Gonzalez Gil (2016), manifiesta en su conferencia: "La extensión universitaria: promotora del cambio, la innovación y la transformación sociocultural" [...] y que "el proyecto es el eje articulador de la gestión del proceso extensionista y elemento clave para dinamizarla y promover las transformaciones".

La URACCAN cuando habla del acompañamiento comunitario intercultural; se refiere a:

un proceso colaborativo, participativo, concertado e inclusivo, sustentado en el diálogo de saberes y haceres, mediante la articulación entre Universidad-Comunidad-Sociedad, que busca transformaciones estructurales, sociales, económicas, políticas, culturales y ambientales de los sistemas que sustentan las desigualdades en torno a la autonomía y autodeterminación de los pueblos, en pro del desarrollo con identidad y el ejercicio efectivo de las ciudadanías interculturales de género, para la construcción colectiva del buen vivir de los pueblos, el respeto, equilibrio y armonía con la Madre Tierra en el ámbitonacional e internacional (URACCAN, 2018).

Esta función institucional es realizada por los Institutos y Centros de Investigación en articulación con las comunidades de aprendizajes: docencia, creación y recreación de conocimientos (investigación) con el objetivo de aportar a la consolidación de las buenas prácticas, saberes y haceres de los pueblos afrodescendientes, indígenas, mestizos y comunidades de la Costa Caribe de Nicaragua y Abya Yala (Latinoamérica), en pro del fortalecimiento de su autonomía, la construcción colectiva del desarrollo con identidad y las ciudadanías interculturales de género que, se materializan mediante el desarrollo con identidad, el buen vivir, el vivir bien y vivir con dignidad.

Los principios fundamentales que la URACCAN ha definido en relación al acompañamiento comunitario intercultural son: i) Es colaborativa, intercultural y solidaria, ii) Desarrolla la perspectiva intercultural de género, iii) Es permanente, flexible y genera cambios, iv) Es holística, multidimensional, v) Articula las funciones de la universidad y los actores institucionales, vi) Recrea conocimientos, saberes y prácticas de los pueblos y la sociedad.

\section{Desafíos del Acompañamiento Comunitario Intercultural}

En el proceso de reflexión al abordar la relación entre sociedad, multiculturalidad, interculturalidad y universidad, se nos presentan algunas interrogantes relativas, a que ¿Si la llamada extensión universitaria en términos genéricos para las Instituciones de Educación Superior (IES), qué es el acompañamiento comunitario intercultural para la URACCAN; cómo esa función que posibilita la articulación en términos de la transformación sociocultural de los pueblos; cuáles son esos grandes desafíos que ella tiene en la vida universitaria? Máxime si la abordamos desde la perspectiva de la multiculturalidad presente en la región y las relaciones interculturales que queremos construir para poder gozar de una sociedad donde todas y todos nos sintamos a gusto.

Estos desafíos los podemos ver en las dimensiones institucionales, en la relación con el Estado y en el acompañamiento a los pueblos.

En la dimensión institucional, los podemos señalar a partir de las siguientes premisas:

1. El cambio de percepción en su conceptualización de extensión, vínculo o proyección social, por ser parte inherente de la comunidad y que con ellos se trabaja con, por y en la comunidad en su aspecto genérico. 
2. El acompañamiento comunitario intercultural desde la universidad debe ser una forma de aprender, integrada a un nuevo acto educativo a lo largo de toda la formación de los estudiantes.

3. Se crea e impulsa un modelo de acompañamiento comunitario intercultural que claramente se refleje en los sectores poblacionales histórica y actualmente vulnerables, invisibilizados y necesitados, sin caer en un carácter hegemónico y asistencialista, a fin de que la comunidad universitaria asuma procesos encaminados a la solución de problemas concretos y que influyan en la transformación social inclusiva, participativa, emancipadora y anticolonialista de nuestros pueblos en un sentido de igualdad, equidad, inclisividad, pertinencia y justicia social.

4. Se institucionalizan recursos humanos, tecnológicos, materiales y financieros para dar respuesta a las necesidades internas de la universidad; pero, esto debe partir de una declaración expresa del modelo de universidad, su proyecto institucional, políticas, reglamentos y manuales que recojan el espiritu de esta función al servicio de la sociedad multicultural, donde se vean explícitos los procesos de planificación, implementación, monitoreo y evaluación de su impacto.

5. El involucramiento colaborativo intercultural de los distintos estamentos de la universidad (investigadores, docentes, estudiantes, personal de apoyo), para visibilizar la articulación universitaria en sus distintas funciones sustanciales, para dar cabida a la responsabilidad institucional expresa en su marco filosófico, epistemológico y transformador.

6. La comunicación, difusión de su implementación y resultados, para que estos sirvan de nutrientes a los procesos de las comunidades de aprendizajes, la creación, recreación de los sistemas de conocimientos, saberes y haceres, mediante la producción de libros, textos, manuales, guías, audiovisuales, entre otros.

7. Recrear los procesos metodológicos del acompañamiento comunitario intercultural, como aprendizajes que nutran nuestros modelos de universidades en el cumplimiento de nuestra razón de ser, la transformación social.

La dimensión en relación con el Estado, los términos de los desafios de la extensión universitaria, se debe referir a:

1. Coadyuvar a la construcción de políticas públicas, que tengan como marco de referencia los abordajes visionarios de las diferencias existentes en términos de identidad, cultura, espiritualidad, cosmovisión, sistemas sociopolítcios, económicos, relacionalidad de los pueblos con la Madre Tierra.

2. Ser la herramienta, el mecanismo de la implementación de políticas públicas inclusivas, pertinentes, equitativas, agenciadoras y transformadoras que redunden en el desarrollo con identidad, el buen vivir de los pueblos y la construcción de ciudadanías interculturales y no sociedades multiculturales. Esto a través del impulso de líneas de acción que vayan en torno a la investigación para el desarrollo, la innovación, la cultura de paz, la gobernanza, la educación intercultural, la diversidad ecológica, humana y natural.

La dimensión del acompañamiento comunitario intercultural de los pueblos, los desafios están encaminados a poder:

1. Impulsar el fortalecimiento de las capacidades organizativas y acompañamiento en las acciones para la promoción, implementación de la gobernanza, cultura de paz, el buen vivir, la sustentabilidad-sostenibilidad en las comunidades-sociedades como una contribución al desarrollo territorial (comunidad, territorio, municipio, región, país). 
2. Impulsar creativamente la revitalización de los conocimientos, saberes y haceres de los pueblos, con especial reconocimiento en su pervivencia natural y social, enfocándonos en la definición, construcción y promoción de políticas institucionales que respeten, promocionen y potencien la propiedad intelectual colectiva para el usufructo y desarrollo de los pueblos.

3. Reconceptualizar el rol de extensionistas, ser y trascender desde la universidad como un agente comunitario que revitalize, promueva y fortalezca a través de la colaboración intercultural, las dinámicas socioculturales, políticas, ambientales y económicas de los pueblos para que se visibilice sus aportes al desarrollo de los países en forma global.

\section{Conclusiones}

La relación entre sociedad, multiculturalidad, interculturalidad y los desafios del acompañamiento comunitario intercultural, esta dada desde la manera en que se conciben, planifican, implementan y evaluan las acciones institucionales de las universidades con la colaboración participativa de los distintos pueblos.

Si el multiculturalismo, es sólo la "convivencia" de culturas; la interculturalidad propugna por cambios profundos y significativos que lleven a desarrollar la capacidad de reconocer las desigualdades que producen las relaciones de poder existentes en nuestras sociedades. Según Dixon (2019); ese "reconocimiento debe conllevar a acciones profundas y transformadoras para lograr cambios significativos en la vida de mujeres y hombres de los diferentes pueblos"5.

Las acciones institucionales interculturales deben de apuntar hacia un tránsito inmediato de lo multicultural a lo intercultural, como ese proceso de relación e intercambio cultural en igualdad de condiciones para cada pueblo, desde donde se construyan nuevos conocimientos, saberes y haceres que redunde en la construcción de una nueva sociedad.

Son muchos los desafíos que las IES tenemos en la construcción de sociedades justas, equitativas, inclusivas, interculturales; por lo que debemos trabajar con una visión holística de nuestros entornos, donde las necesidades y prioridades de los pueblos sean el horizonte de nuestro accionar, lo cual llevaría al camino y proposito inicial de las universidades: constituirse en el foro para buscar soluciones que aquejan a la sociedad.

En este marco hay que ver al acompañamiento comunitario como el proceso, instrumento y mecanismo que permite a través de sus programas, proyectos y acciones transformar la realidad.

Las instituciones de educación superior, a través de sus funciones y ámbitos de su quehacer, debe reafirmar y fortalecer el carácter pluricultural, multiétnico y multilingüe de nuestros países y de nuestra región"6.

\section{Agradecimientos}

Esta publicación obtuvo el financiamiento de: El Fondo de Asistencia Internacional de los Estudiantes y Académicos Noruegos (SAIH).

\section{Lista de referencias}

Dixon Berna (2019). Seminario Universidad para la Paz. UNAN, Managua,

Henríquez G. P. (2018). Tendencias de la Educación Superior en América Latina y el Caribe. IESALC-UNESCO, Universidad de Córdoba, Córdoba, Argentina.

\footnotetext{
5 Dixón, Berna, 2019; UNAN, Managua, Seminario Universidad para la Paz.

6 IESALC-UNESCO, 2008; Declaración II Conferencia Regional, Cartagena de Indias, Colombia.
} 
Hooker B. Alta (2019). Conferencia Magistral "La Interculturalidad: una propuesta para el desarrollo sostenible de los pueblos originarios y afrodescendientes". Universidad Politécnica de Nicaragua. Managua, Nicaragua, 15 de noviembre.

IESALC-UNESCO, 1996, Conferencia Regional de Educación Superior, La Habana, Cuba.

IESALC-UNESCO (2008). Conferencia Regional de Educación Superior, Cartagena de Indias, Colombia.

IESALC-UNESCO (2018). Conferencia Regional de Educación Superior. Córdoba, Argentina.

Mato D. (2017). Educación Superior y Pueblos Indígenas y Afrodescendientes, en América Latina. Políticas y prácticas de inclusión, democratización e interculturalización. Editorial UNTREF, Córdoba, Argentina.

XIV Congreso Latinoamericano de Extensión Universitaria y el 2do. Congreso Centroamericano de Compromiso Social convocados respectivamente por la Unión Latinoamericano de Extensión Universitaria (ULEU) y el Consejo Superior de Universidades Centroamericanas (CSUCA) y con el auspicio del Consejo Nacional de Universidades de Nicaragua (CNU), Managua, Nicaragua, 05 al og de Junio 2017.

URACCAN (2018). Política de Acompañamiento Comunitario Intercultural. Regiones Autónomas de la Costa Caribe Nicaragüense, Nicaragua.

URACCAN (2015). Plan Estratégico Institucional 2015-2019. Regiones Autónomas de la Costa Caribe Nicaragüense, Nicaragua. 